Submitted to:

Twenty Eighth International Symposium on Combustion

University of Edinburgh, Scotland

July30 - August 4, 2000

\title{
THEORETICAL KINETIC ESTIMATES FOR THE RECOMBINATION OF HYDROGEN ATOMS WITH PROPARGYL AND ALLYL RADICALS
}

\section{LAWRENCE B. HARDING ${ }^{1}$ AND STEPHEN J. KLIPPENSTEIN ${ }^{2}$}

The submitted manuscript has been created by the University of Chicago as Operator of Argonne National Laboratory ("Argonne") under Contract No. W-31-109-ENG-38 with the U.S. Department of Energy. The U.S. Government retains for itself, and others acting on its behalf, a paid-up, nonexclusive, irrevocable worldwide license in said article to reproduce, prepare derivative works, distribute copies to the public, and perform publicly and display publicly, by or on behalf of the Government.

\author{
${ }^{1}$ Chemistry Division, \\ Argonne National Laboratory, \\ Argonne, IL, 60439, USA \\ ${ }^{2}$ Chemistry Department, \\ Case Western Reserve University, \\ Cleveland, $\mathrm{OH}, 44106-7078$, USA
}

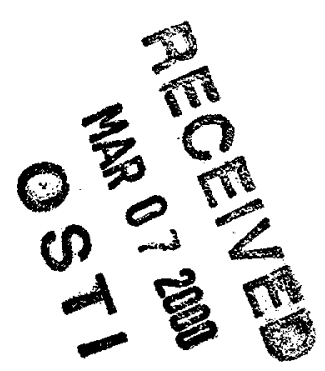

Fax: (216)368-3006

E-mail: sjk5@po.cwru.edu

Colloquium: Reaction Kinetics of Combustion

Word Count: 5357 Total words $=3957$ words +6 Figures * 200 words +1 Table*200 words

Submitted for Oral Presentation

Abstract

$A b$ initio quantum chemical simulations are coupled with variational transition state theory in estimating rate constants for the $\mathrm{H}+\mathrm{C}_{3} \mathrm{H}_{3}$ and $\mathrm{H}+\mathrm{C}_{3} \mathrm{H}_{5}$ recombination reactions. The energy of interaction between the $\mathrm{H}$ atom and each of the radicals is evaluated at the $\mathrm{CAS}+1+2$ level for the range of separations and relative orientations spanning the transition state region. An analytic representation of these interaction energies is then implemented in variable reaction coordinate transition state theory calculations of the high pressure limit recombination rate constant for temperatures ranging from 200 to $2000 \mathrm{~K}$. For the propargyl reaction the overall addition rate is separated into contributions correlating with the initial formation of allene and propyne. These theoretical results are compared with the available experimental data as well as with corresponding theoretical estimates for the $\mathrm{H}_{+} \mathrm{C}_{2} \mathrm{H}_{3}$ and $\mathrm{H}+\mathrm{C}_{2} \mathrm{H}_{5}$ reactions. The $\mathrm{H}+$ propargyl and $\mathrm{H}+\mathrm{allyl}$ total recombination rates are remarkably similar, with both being greater than the H+vinyl and $\mathrm{H}+\mathrm{ethyl}$ rates, due to the presence of twice as many addition channels. 


\section{DISCLAIMER}

This report was prepared as an account of work sponsored by an agency of the United States Government. Neither the United States Government nor any agency thereof, nor any of their employees, make any warranty, express or implied, or assumes any legal liability or responsibility for the accuracy, completeness, or usefulness of any information, apparatus, product, or process disclosed, or represents that its use would not infringe privately owned rights. Reference herein to any specific commercial product, process, or service by trade name, trademark, manufacturer, or otherwise does not necessarily constitute or imply its endorsement, recommendation, or favoring by the United States Government or any agency thereof. The views and opinions of authors expressed herein do not necessarily state or reflect those of the United States Government or any agency thereof. 


\section{DISCLAIMER}

\section{Portions of this document may be illegible in electronic image products. Images are produced from the best available original document.}




\section{Introduction}

It has been widely suggested that the relatively high concentrations (in the combustion environment) of resonantly stabilized radicals, such as the propargyl radical, is the result of their comparatively low rates of reaction [1]. In this work we examine the reaction kinetics for the recombination of $\mathrm{H}$ atoms with two such resonantly stabilized radicals; the allyl radical $\left(\mathrm{CH}_{2} \mathrm{CHCH}_{2}\right)$ and the propargyl radical $\left(\mathrm{CHCCH}_{2}\right)$. The allyl radical, with its two degenerate resonance structures, is a prototypical resonantly stabilized radical. The propargyl radical also has two dominant resonance structures but, unlike allyl, the two structures are not equivalent.

There is considerable recent interest in the rates of propargyl radical reactions due to the postulated role of propargyl radicals in the soot formation process, and particularly the initial formation of benzene [1]. In spite of this interest, the available experimental data for both the H+propargyl [2-4] and the H+allyl [5] reactions is quite limited. The present coupling of high-level quantum chemical simulations with sophisticated transition state theory evaluations should provide quantitatively meaningful a priori estimates for the kinetics of these two reactions. Comparison with related theoretical results for the $\mathrm{H}+$ vinyl [6] and $\mathrm{H}+$ ethyl [7] reactions allows for a reasonably direct examination of the effects of resonance stabilization on the kinetics.

For the propargyl radical there are two distinct addition products, allene $\left(\mathrm{CH}_{2} \mathrm{CCH}_{2}\right)$ and propyne $\left(\mathrm{CH}_{3} \mathrm{CCH}\right)$, formed by attack on different ends of the propargyl radical. For allyl, the only stable addition product is propene $\left(\mathrm{CH}_{3} \mathrm{CHCH}_{2}\right)$, which can be formed by attack on either end. Both radicals are planar, and attack can occur from either above or (equivalently) below the plane of the radical. Thus for allyl there are four distinct pathways all of which are related by symmetry. For propargyl, there are also four distinct pathways, which can be divided into two symmetry related pairs, one pair leading to allene and the other to propyne.

Here, the interaction of the $\mathrm{H}$ atom with each of the radicals for each of the addition paths is first characterized with high level $a b$ initio quantum chemical simulations. The resulting analytical potentials are then implemented in variable reaction coordinate transition state theory based estimates for the high pressure recombination kinetics. The results of these calculations are directly compared with the available experimental data. 


\section{Potential Surface Calculations}

\section{Methodology}

All of the electronic structure results reported here are from multi-reference configuration interaction (MRCI) calculations employing orbitals optimized using the complete active space, self-consistent field (CASSCF) methodology. In these calculations, the CASSCF reference wavefunction consists of four active orbitals (the hydrogen 1s orbital and three $\pi$ orbitals on the hydrocarbon fragment) and four active electrons. This is the minimum necessary to correctly describe the breaking $\mathrm{CH}$ bond and the resonance of the hydrocarbon radical. The effects of higher order excitations were tested using a multi-reference Davdison correction. The basis set used in most calculations was the correlation-consistent, polarized valence double zeta (cc-pvdz) basis set of Dunning [8-10]. To estimate the error associated with the use of this relatively small basis set, internally contracted, multi-reference $\mathrm{CI}$ calculations were carried out along the association paths for $\mathrm{H}+\mathrm{C}_{3} \mathrm{H}_{3}$ using the cc-pvtz basis set. The un-contracted $\mathrm{CI}$ calculations were carried out using the COLUMBUS package of codes [11]. The internally contracted $\mathrm{CI}$ calculations were done using the MOLPRO package [12-16].

\section{Results}

Characterization of the potential surface for the association reactions was accomplished by mapping out three dimensional grids, in polar coordinates, corresponding to motion of the hydrogen atom reactant around the rigid hydrocarbon radicals. These surfaces were fit using techniques described previously in calculations on $\mathrm{H}+\mathrm{C}_{2} \mathrm{H}_{3}$ [6] and $\mathrm{H}+\mathrm{C}_{2} \mathrm{H}_{5}$ [7].

A two dimensional slice of the three dimensional, $\mathrm{H}+\mathrm{C}_{3} \mathrm{H}_{3}$ surface is shown in Figure 1. From this plot it can be seen that there are four barrierless attractive valleys through which association reactions can take place; two, symmetry related paths, for attack on the $\mathrm{CH}$ end of $\mathrm{C}_{3} \mathrm{H}_{3}$ and two for attack on the $\mathrm{CH}_{2}$ end. Recall that the ground state, electronic wavefunction of propargyl is an un-symmetrical resonance between a structure having two carbon-carbon double bonds (with the radical orbital on the $\mathrm{CH}$ end) and one having one carbon-carbon single bond and one carbon-carbon triple bond (with the radical on the $\mathrm{CH}_{2}$ end). The $\mathrm{C}_{2 \mathrm{v}}$ equilibrium structure of propargyl implies that the single bond - triple bond structure is the dominant resonance structure. Figure 1 shows that the paths for attack on the $\mathrm{CH}_{2}$-side are significantly more attractive and wider (in this in-plane coordinate) than the paths for attack on the $\mathrm{CH}$-side, i.e., there is, not surprisingly, an energetic preference for attack of the dominant resonance structure. In Figure 2 we show two perpendicular slices through this potential surface, centered on each of the terminal carbon atoms. From this plot it can be seen that the $\mathrm{CH}$-side paths are somewhat wider in this coordinate than the $\mathrm{CH}_{2-}$ 
side paths. This is caused by non-bonded repulsions between the incoming $\mathrm{H}$ atom and the two $\mathrm{CH}$ bonds on the $\mathrm{CH}_{2}$-side.

For the H+allyl reaction, the calculations show four symmetry related pathways for barrierless reaction (the hydrogen can attack either terminal carbon from either above or below the plane of the radical). The calculations also predict that approach of the central carbon is repulsive (as was found for $\mathrm{H}+\mathrm{C}_{3} \mathrm{H}_{3}$ ).

To better compare the relative attractive-ness of the association paths we optimized minimum energy paths (MEP) for both $\mathrm{H}+$ allyl and $\mathrm{H}+$ propargyl. In these calculations the $\mathrm{C}-\mathrm{H}$ distance is kept fixed at a number of different values and the two angular coordinates are optimized to find the orientation with the lowest energy (again the geometries of the hydrocarbon radical fragments are kept fixed). Plots of the energy along these MEP's are shown in Figure 3 along with comparable calculations on $\mathrm{H}^{+} \mathrm{C}_{2} \mathrm{H}_{3}$ and $\mathrm{H}+\mathrm{C}_{2} \mathrm{H}_{5}$. From this plot it can be seen that the H+allyl MEP lies between the two H+propargyl MEP's. All of the H+resonance stablized radical MEP's are predicted to be significantly less attractive than the MEP's for the non-resonance stabilized radicals, $\mathrm{C}_{2} \mathrm{H}_{3}$ and $\mathrm{C}_{2} \mathrm{H}_{5}$.

The MEP's for the two reactions, $\mathrm{H}+\mathrm{C}_{3} \mathrm{H}_{3} \rightarrow \mathrm{CH}_{2} \mathrm{CCH}_{2}$ and $\mathrm{H}+\mathrm{C}_{3} \mathrm{H}_{3} \rightarrow \mathrm{CH}_{3} \mathrm{CCH}$, were recalculated using the larger cc-pvtz basis set. The cc-pvtz MEP's were found to be slightly more attractive than the cc-pvdz MEP's. The cc-pvdz potential surface was corrected for this deficiency as follows. At each point the distance between the hydrogen atom and each of the terminal carbon atoms is determined. Two corrections are then calculated, one using the difference between the cc-pvdz and cc-pvtz MEP's for addition to the $\mathrm{CH}_{2}$ end and the distance between the $\mathrm{H}$ atom and the $\mathrm{CH}_{2}$ carbon. The second correction uses the difference between the two MEP's for addition to the $\mathrm{CH}$ end and the distance between the $\mathrm{H}$ atom and the $\mathrm{CH}$ carbon. Which ever of these two corrections is largest is then used to correct the cc-pvdz surface at that point.

\section{Transition State Theory}

For barrierless reactions such as the radical-radical recombination reactions of interest here, the optimal form for the transition state dividing surface varies considerably with energy $(E)$ and with total angular momentum $(J)$. At low $E$ and low $J$ the transition state lies at large interfragment separations and the optimal reaction coordinate is simply the separation between the centers-of-mass of the two radicals. At higher $E$ and/or $J$ the transition state moves in to shorter separations where the optimal transition state dividing surface correlates, at least qualitatively, with a fixed separation between the centers of the two orbitals involved in the incipient bond. 
In this work, both the definition and location of the transition state dividing surface are variationally optimized within the set of surfaces defined by a fixed separation between the $\mathrm{H}$ atom and a pivot point connected to the propargyl or allyl radical. Choosing the pivot point as the center-of-mass of the radical yields a form appropriate for low $E$ and $J$. Alternatively, choosing the pivot point along the axis of one of the lone pair orbitals of the radical provides a dividing surface that is appropriate for high $E$ and/or high $J$. Minimization of the transition state partition function with respect to both the location of the radical pivot point and the separation between the $\mathrm{H}$ atom and the pivot point then provides the present variable reaction coordinate transition state theory estimate for the rate constant.

For the propargyl radical, there are four distinct reaction paths for the addition, corresponding to the addition of the $\mathrm{H}$ atom from either above or below the radical plane to either the $\mathrm{CH}$ or the $\mathrm{CH}_{2}$ side of the radical. The presence of strongly repulsive regions separating each of these channels, and the extension of these repulsive regions out to fairly large separations, suggests that it is appropriate to optimize separate dividing surfaces for each of these channels. The $\mathrm{CH}$ (allene) and $\mathrm{CH}_{2}$ (propyne) addition channels are distinguished here by the angle between the incoming $\mathrm{H}$ atom and the $\mathrm{CC}$ bond from the central $\mathrm{C}$ to the methylene C. Angles of greater than $90^{\circ}$ are assumed to correlate with allene while values of less than $90^{\circ}$ are assumed to correlate with propyne.

A grid based procedure is employed in the variational minimization at the $E / J$ resolved level for each of these channels. The grid of radical fixed-point locations is defined in terms of the vector from the fixed point to the $\mathrm{C}$ atom of the incipient bond. This vector is taken to lie in the plane perpendicular to that of the radical, but passing through the CCC axis, and is defined in terms of its length $d$ and angle $\theta$ relative to the $\mathrm{CC}$ axis. Sample calculations suggested that two distinct sets of $(d, \theta)$ should be considered. The first set is centered about $(1.2,0)$ and effectively yields the center-of-mass reaction coordinate. The second set includes $d$ values ranging from 0.0 to 1.25 and angles ranging from about 70 to 110 . This second set samples fixed points in the neighborhood of the radical orbital. The first set of fixed point locations is coupled with a grid of $\mathrm{H}$ atom to radical fixed-point separations $\mathrm{R}$ ranging from about 5.0 to about 3.25 (1), with a 0.25 (1) spacing. The second set of fixed-point locations is coupled with a grid of $R$ ranging from about 3.0 to $1.75(1)$, again with a 0.25 (1) spacing. The VARIFLEX software package [17] was used in evaluating the number of available states, $N_{E J}(R, d, \theta)$, [i.e., the $\mathrm{E} / \mathrm{J}$ resolved transition state partition function] for each point in this grid, and in performing the Boltzmann weighted integral over $E$ and $J$ of the minimum of $N_{E J}$ on the grid to obtain the thermal rate constant. Interestingly, the optimized dividing surfaces were again [6] found to correlate closely with the contours of the radical orbitals.

For the allyl radical there are again four distinct reaction paths, but these are now all symmetrically related. Each of these channels is again well separated by repulsive regions of the potential and so optimized 
dividing surfaces are considered for each channel separately. The procedure employed in these optimizations is closely analogous to that for the propargyl radical and so is not reviewed here.

The variable reaction coordinate formalism [18] employed here, also implements an assumed separation between the internal vibrational modes of the fragments, labeled the conserved modes, and the remaining transitional modes, which vary in character from the reactants to the products. Here, the properties of the conserved modes are assumed to be invariant in the process from reactants to the transition state. Then, their contribution to the transition state partition function cancels the corresponding contribution to the reactant partition function. In reality, both the vibrational frequencies and the optimal geometry will vary somewhat as the fragments approach one another. For example, the $\mathrm{H}$ atom(s) on the $\mathrm{C}$ being attacked by the incoming $\mathrm{H}$ atom will move away from the appoaching $\mathrm{H}$ atom by bending out of the plane of the radical. Concomitant with this change in bending angle(s) will be a change in its vibrational frequency and other related torsional frequencies. However, such variations tend to be of only secondary importance. For example, for the frontside addition channel in the $\mathrm{H}+$ vinyl reaction these two effects yield a correction of only $4 \%$. Thus such variations, which would require substantial additional computational effort, were neglected here.

\section{Results and Discussion}

The present MR-CI based variable reaction coordinate transition state theory results for the H+propargyl high pressure recombination rate constant are plotted in Figure 4. The ratio of the propyne to allene formation rate constants is roughly constant at 1.55 for temperatures up to $1000 \mathrm{~K}$ and then decreases gradually with increasing temperature, reaching a value of 1.45 at $2000 \mathrm{~K}$. This larger magnitude for the propyne formation channel is readily explained on the basis of the greater attractivenss along the MEP (cf. Figure 3), and somewhat larger range of angles for which the interaction is attractive for this channel (cf. Figure 1).

The available experimental results, which are indirectly determined from kinetic modeling of the pyrolysis of toluene [3], and the oxidations of acetylene [2] and propargyl [4], are also plotted in Figure 4. The theoretical predictions are in quantitative agreement with the most recent of these experimental results, which were obtained for a pressure of $6.65 \mathrm{mbar}$ at a temperature of $295 \mathrm{~K}$. Sample master equation simulations of the pressure dependence suggest that the discrepancy of a factor of 3 to 10 with the other experimental results may, at least in part, be due to a deviation from the high pressure limit in these experimental studies. 
The present data could also be used to predict $\mathrm{CH}$ dissociation rates in allene and propyne; for which there is a substantially larger body of experimental data [19]. However, as has been well described by Kiefer et al. [19], the proper modeling of the dissociation process requires a good description of the effect of vibrational anharmonicities on the state density of the complex; which is beyond the scope of the present work. Our own sample master equation calculations employing the present VRC-TST model for the transition state yield similar results to those of Ref. [19]. Thus, we do not pursue here a comparison with dissociation rates.

Related calculations of the $\mathrm{H}+\mathrm{C}_{3} \mathrm{H}_{3}$ recombination rate, employing a potential which does not include the cc-pvtz basis set corrections, yield results which are only 9 to $19 \%$ lower. Similarly, the neglect of the Davidson correction yields results that are only 8 to $20 \%$ lower. The smallness of these two corrections suggests that any inaccuracies in the underlying potential are likely to be of only minor significance.

The present results for the H+allyl recombination rate are plotted in Figure 5. The theoretical predictions are seen to be in quantitative agreement with the fairly direct experimental measurement of Hanning-Lee and Pilling [5]. The predicted rise by a factor of 1.54 from 200 to $2000 \mathrm{~K}$ is identical to that predicted for the H+propargyl reaction. A cc-pvtz calculation of the MEP was not performed for this reaction. However, the near constancy of the effect of this correction on the rates for a variety of reactions led us to simply incorporate a $15 \%$ increase to the rates. The effect of the Davidson correction is also expected to be similar to that for the H+propargyl reaction.

A plot of the VRC-TST predictions for the high pressure recombination rate constants for the $\mathrm{H}+$ propargyl, allyl, vinyl and ethyl reactions is provided in Figure 6. The rate constant for the $\mathrm{H}+\mathrm{C}_{3} \mathrm{H}_{5}$ recombination is remarkably similar to the total recombination rate constant for $\mathrm{H}_{3}+\mathrm{C}_{3} \mathrm{H}_{3}$. Interestingly, the $\mathrm{H}+\mathrm{C}_{3} \mathrm{H}_{5}$ MEP lies roughly midway between those for the two $\mathrm{H}+\mathrm{C}_{3} \mathrm{H}_{3}$ channels. As a result, while the addition to the $\mathrm{CH}_{2}$ side of propargyl occurs at a greater rate than does the addition to one side of allyl, the addition to the $\mathrm{CH}$ side of propargyl occurs at a lower rate. The sum of the rates for the two propargyl channels then adds to be essentially identical to the total allyl rate.

The $\mathrm{H}$ atom recombination rates for the two resonantly stabilized radicals are actually predicted to be greater than those for the vinyl and ethyl radicals. At first glance this seems to contradict the increased attractiveness for the MEP's in the vinyl and ethyl radicals (cf. Figure 3). However, this result is simply an indication that there are essentially four separate addition channels for each of the resonantly stabilized channels, while there are only two (frontside and backside additions) for the vinyl and ethyl radicals. After division by two the rates for the allyl and propargyl radicals are, as expected, significantly smaller than those for the vinyl and ethyl radicals. 


\section{Summary}

The $\mathrm{H}+$ allyl and $\mathrm{H}+$ propargyl recombination reactions have been studied with variable reaction coordinate transition state theory employing interaction potentials obtained from multi-reference configuration interaction calculations. The predictions for the high pressure limit recombination rates were found to be essentially identical for these two reactions. The results for the H+allyl reaction are in good agreement with the only experimental study, while those for the $\mathrm{H}+$ propargyl reaction agree only with those from the most recent experimental study, being considerably greater than other earlier studies. The addition to the $\mathrm{CH}_{2}$ side of the propargyl radical is predicted to occur with a rate that is 1.5 times that for the $\mathrm{CH}$ side.

Table I provides the parameters $A, n$, and $E_{a}$ from modified Arrhenius fits $\left[k=A T^{n} \exp \left(-E_{a} / T\right)\right]$ to the high pressure recombination rates for $\mathrm{H}+$ allyl, $\mathrm{H}+$ propargyl, $\mathrm{H}+$ vinyl, and $\mathrm{H}+\mathrm{ethyl}$. Contrary to initial expectations the high pressure recombination rates for the two resonantly stabilized radicals studied here are actually greater than those for the $\mathrm{H}+\mathrm{vinyl}$ and $\mathrm{H}+\mathrm{ethyl}$ reactions, due to the presence of twice as many addition channels for the resonantly stabilized radicals. Similar findings might be expected for the relative recombination rates with other addition partners.

The observation of elevated concentrations of these radicals thus appears to be simply the result of greater rates of formation, i.e., their stabilization energy should correlate with decreased production thresholds relative to other related channels. At lower pressures these decreased production thresholds might also affect the ordering of various effective recombination rates. In particular, the redissociation of the addition products back to reactants would be expected to be greater for the resonantly stabilized radicals due to their lower binding energy, and thus the effective loss rates would be smaller. Of course such a pressure dependent effect would also depend quite strongly on the lifetime (and correspondingly the size) of the complex.

\section{Acknowledgments}

This research was supported by the U.S. Department of Energy, Office of Basic Energy Sciences, Division of Chemical Sciences, under Contracts W-31-109-ENG-38 (LBH) and DE-FG-02-98ER14902 (SJK). 


\section{REFERENCES}

(1) Miller, J. A., and Melius, C. F., Combust. Flame 91:21-39 (1992).

(2) Homann, K. H., and Wellmann, Ch., Ber. Bunsenges. Phys. Chem. 87:609-616 (1983); and references cited therein.

(3) Braun-Unkhoff, M., Frank, P., and Just, Th., in Twenty-Second Symposium (International) on Combustion, The Combustion Institute, 1988, pp. 1053-1061.

(4) Atkinson, D. B., and Hudgens, J. W., J. Phys. Chem. A, 103:4242-4252 (1999).

(5) Hanning-Lee, M. A., and Pilling, M. J., Int. J. Chem. Kinet. 24:271-278 (1992).

(6) Klippenstein, S. J., and Harding, L. B., Phys. Chem. Chem. Phys. 1:989-997 (1999).

(7) Harding, L. B., and Klippenstein, S. J., in Twenty-Seventh Symposium (International) on Combustion, The Combustion Institute, Pittsburgh, 1998, pp. 151-157.

(8) Dunning Jr., T. H., J. Chem. Phys. 90:1007-1023 (1989).

(9) Kendall, R. A., Dunning Jr., T. H., and Harrison, R. J., J. Chem. Phys. 96:6796-6806 (1992).

(10) Woon, D. E., and Dunning Jr., T. H., J. Chem. Phys. 98:1358-1371 (1993).

(11) Shepard, R., Shavitt, I., Pitzer, R. M., Comeau, D. C., Pepper, M., Lischka, H., Szalay, P. G., Ahlrichs, R., Brown, F. B., and Zhao, J.-G., Int. J. Quantum Chem. S22:149-165 (1988).

(12) MOLPRO is a package of ab initio programs written by Werner, H.-J. and Knowles, P. J. with contributions from Almlof, J., Amos, R. D., Berning, A., Cooper, D. L., Deegan, M. J. O., Dobbyn, A. J., Eckert, F., Elbert, S. T., Hampel, C., Lindh, R., Lloyd, A. W., Meyer, W., Nicklass, A., Peterson, K., Pitzer, R., Stone, A. J., Taylor, P. R., Mura, M. E., Pulay, P., Schutz, M., Stoll, H., Thorsteinsson, $T$.

(13) Werner, H.-J. and Knowles, P.J. J. Chem. Phys. 82:5053-5063 (1985).

(14) Knowles, P.J., and Werner, H.-J. Chem. Phys. Lett. 115:259-267 (1985).

(15) Werner, H.-J. and Knowles, P.J. J. Chem. Phys. 89:5803-5814 (1988).

(16) Knowles, P.J., and Werner, H.-J. Chem. Phys. Lett. 145:514-522 (1988).

(17) Klippenstein, S. J., Wagner, A. F., Dunbar, R. C., Wardlaw, D. M., and Robertson, S. H., VARIFLEX, Version 1.0, (July 1999).

(18) Klippenstein, S. J., J. Phys. Chem. 98:11459-11464 (1994).

(19) Kiefer, J. H., Mudipalli, P. S., Sidhu, S.S., Kern, R. D., Jursic, B. S., Xie, K., and Chen, H., J. Phys. Chem. $A, 101: 4057-4071$ (1997) and references cited therein. 


\section{Figure Captions:}

Fig. 1. Two dimensional cut of the three dimensional surface for the $\mathrm{H}+\mathrm{C}_{3} \mathrm{H}_{3} \rightarrow \mathrm{C}_{3} \mathrm{H}_{4}$ association reaction. The plotting plane is perpendicular to the plane of the $\mathrm{C}_{3} \mathrm{H}_{3}$ radical and bisects the $\mathrm{HCH}$ angle. Solid contours are positive, dashed contours negative and the zero energy contour (defined to be the energy of the $\mathrm{H}+\mathrm{C}_{3} \mathrm{H}_{3}$ ) is shown with a heavy solid line. The contour increment is $2 \mathrm{kcal} / \mathrm{mole}$ and all distances are shown in atomic units (one atomic unit $=0.52918 \AA$ ).

Fig. 2. Two dimensional cuts of the three dimensional surface for the $\mathrm{H}+\mathrm{C}_{3} \mathrm{H}_{3} \rightarrow \mathrm{C}_{3} \mathrm{H}_{4}$ association reaction. The plotting planes are perpendicular to the CCC axis. The plotting plane for the upper plot contains the $\mathrm{CH}_{2}$ carbon, while that of the lower plot contains the $\mathrm{CH}$ carbon. The other ploting conventions are as in Figure 1.

Fig. 3. MEP energies for the reactions: $\mathrm{H}+\mathrm{C}_{3} \mathrm{H}_{5} \rightarrow \mathrm{C}_{3} \mathrm{H}_{6}$ (solid), $\mathrm{H}+\mathrm{C}_{3} \mathrm{H}_{3} \rightarrow \mathrm{CH}_{2} \mathrm{CCH}_{2}$ (upper, dashed), $\mathrm{H}+\mathrm{C}_{3} \mathrm{H}_{3} \rightarrow \mathrm{CH}_{3} \mathrm{CCH}$ (lower, dashed), $\mathrm{H}+\mathrm{C}_{2} \mathrm{H}_{3} \rightarrow \mathrm{C}_{2} \mathrm{H}_{4}$ (dash-dot), and $\mathrm{H}+\mathrm{C}_{2} \mathrm{H}_{5} \rightarrow \mathrm{C}_{2} \mathrm{H}_{6}$ (dot).

Fig. 4. Plot of the high pressure recombination rate constant versus temperature for $\mathrm{H}+\mathrm{C}_{3} \mathrm{H}_{3}$, including the separate contributions corresponding to the propyne and allene product channels.

Fig. 5. Plot of the high pressure recombination rate constant versus temperature for $\mathrm{H}+\mathrm{C}_{3} \mathrm{H}_{5}$.

Fig. 6. Plot of the high pressure recombination rate constant versus temperature for $\mathrm{H}_{+} \mathrm{C}_{3} \mathrm{H}_{5}$ (solid), $\mathrm{H}+\mathrm{C}_{3} \mathrm{H}_{3}$ (upper, dashed), $\mathrm{H}+\mathrm{C}_{2} \mathrm{H}_{3}$ (dash-dot), and $\mathrm{H}+\mathrm{C}_{2} \mathrm{H}_{5}$ (dot). The two lower dashed lines are for the propyne and allene (lowest) channels of the $\mathrm{H}+\mathrm{C}_{3} \mathrm{H}_{3}$ recombination. 
Table I: Modified Arrhenius parameters for $\mathrm{H}+$ radical high pressure recombination rates.

\begin{tabular}{|l|l|l|l|}
\hline Reaction & $\mathrm{A}\left(10^{-11} \mathrm{~cm}^{3} \mathrm{~s}^{-1}\right)$ & $\mathrm{n}$ & $\mathrm{E}_{\mathrm{a}}(\mathrm{K})$ \\
\hline $\mathrm{H}+\mathrm{C}_{3} \mathrm{H}_{5} \rightarrow$ products & 5.804 & 0.2363 & -25.90 \\
\hline $\mathrm{H}+\mathrm{C}_{3} \mathrm{H}_{3} \rightarrow$ products & 4.520 & 0.2711 & -42.24 \\
\hline $\mathrm{H}+\mathrm{C}_{3} \mathrm{H}_{3} \rightarrow$ propyne & 3.710 & 0.2298 & -24.26 \\
\hline $\mathrm{H}+\mathrm{C}_{3} \mathrm{H}_{3} \rightarrow$ allene & 1.139 & 0.3320 & -69.06 \\
\hline $\mathrm{H}+\mathrm{C}_{2} \mathrm{H}_{3} \rightarrow$ products & 4.279 & 0.2724 & -37.56 \\
\hline $\mathrm{H}+\mathrm{C}_{2} \mathrm{H}_{5} \rightarrow$ products & 10.38 & 0.1017 & 15.84 \\
\hline
\end{tabular}




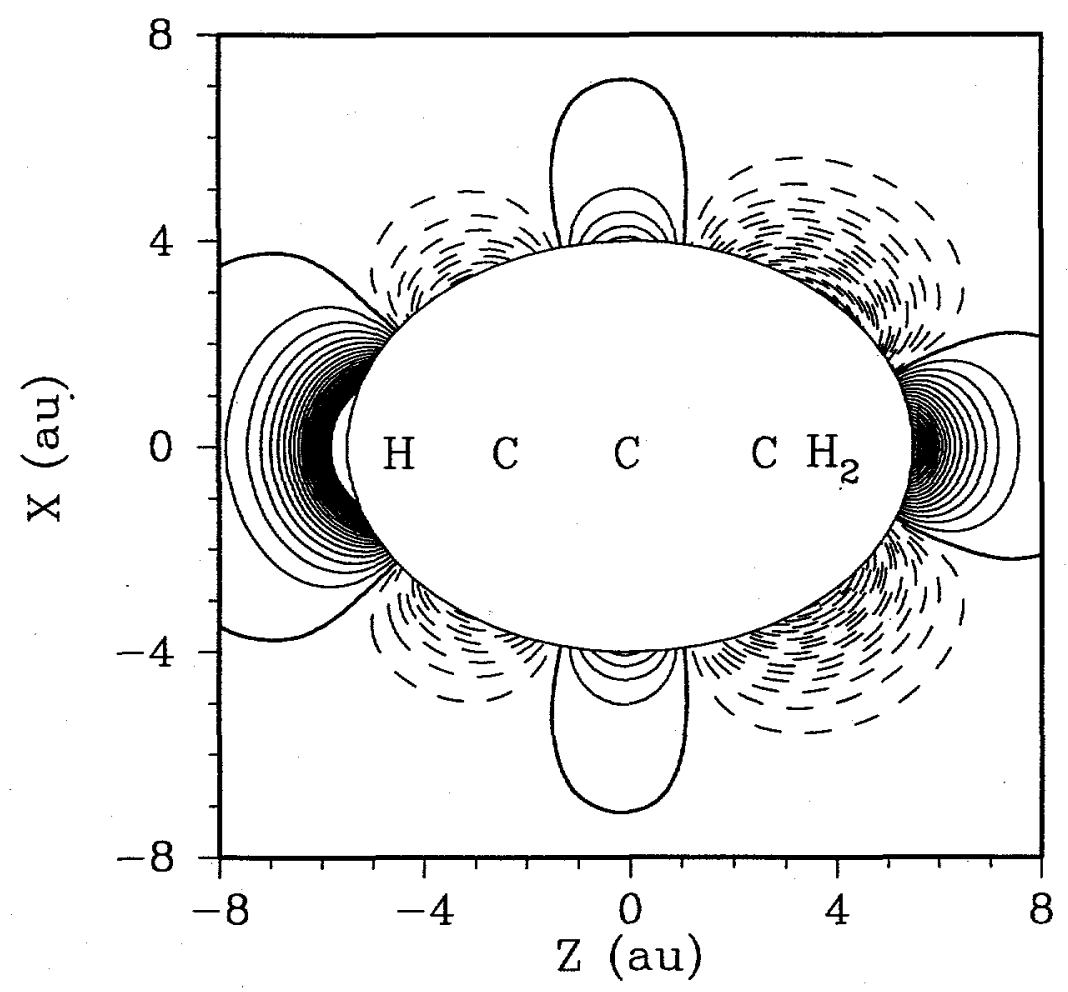



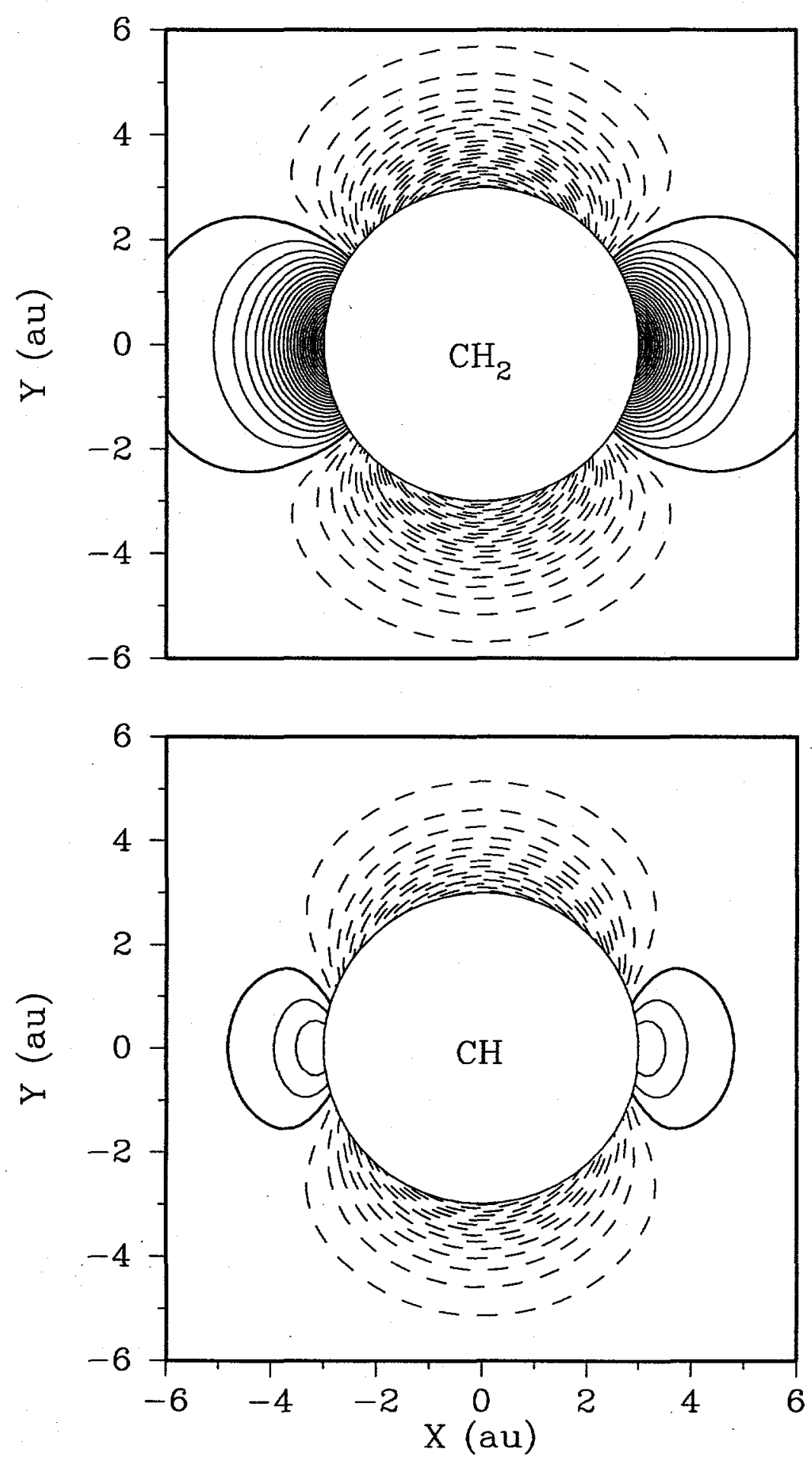


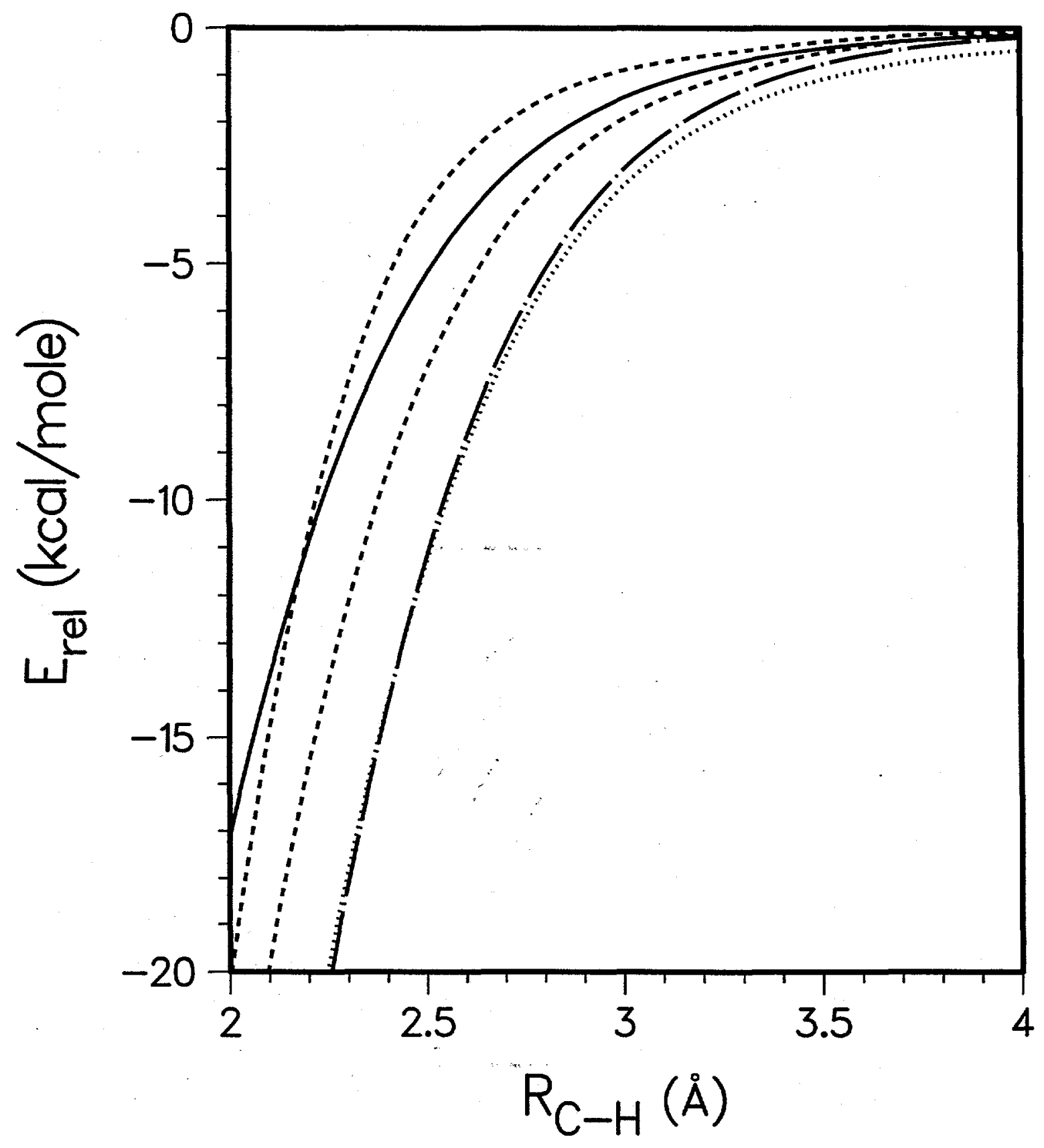


(y) $\perp$

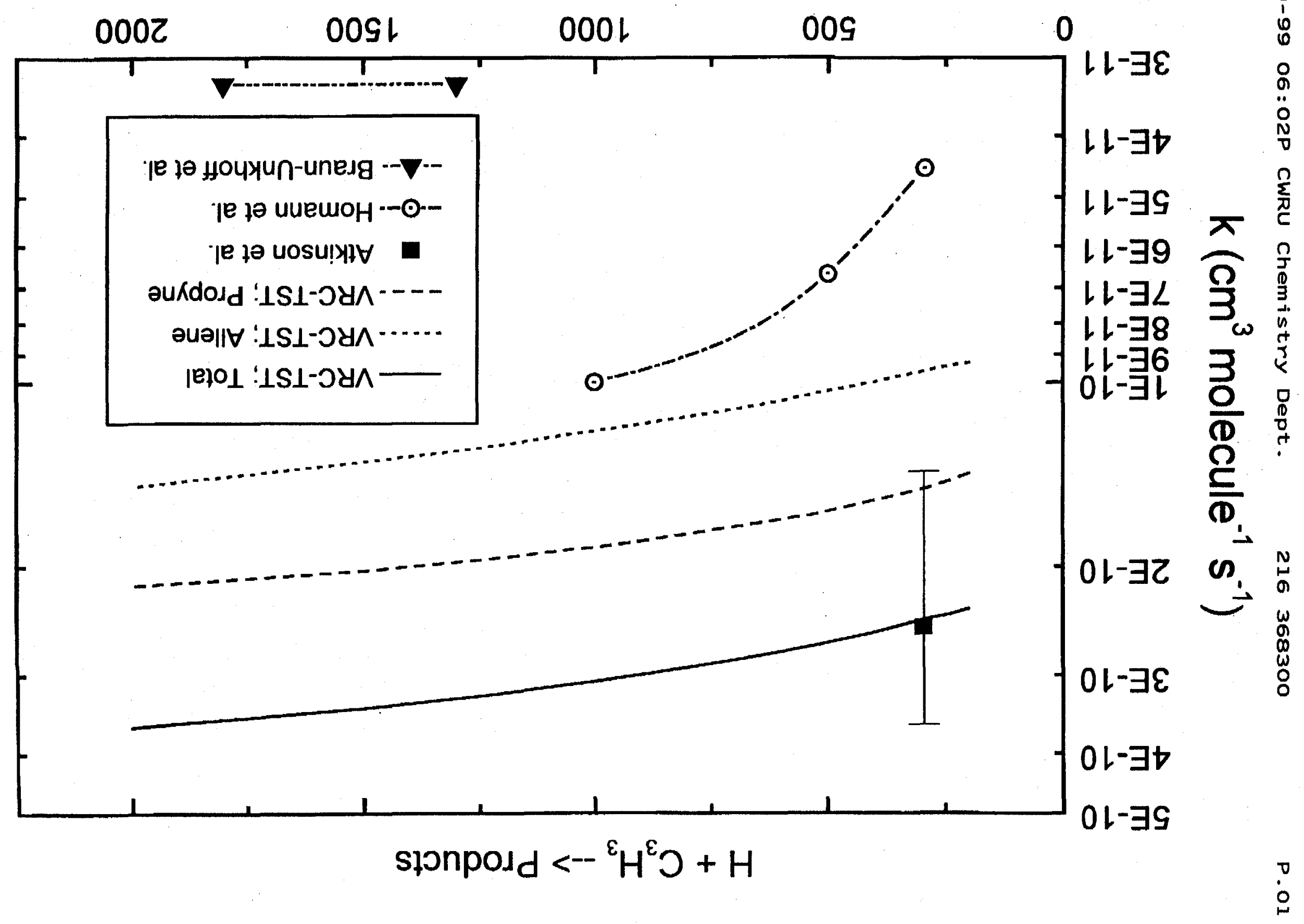


Dec-1.0-99 06:03P CWRU Chemistry Dept.

$\mathrm{k}\left(\mathrm{cm}^{3}\right.$ molecule $\left.^{-1} \mathrm{~s}^{-1}\right)$

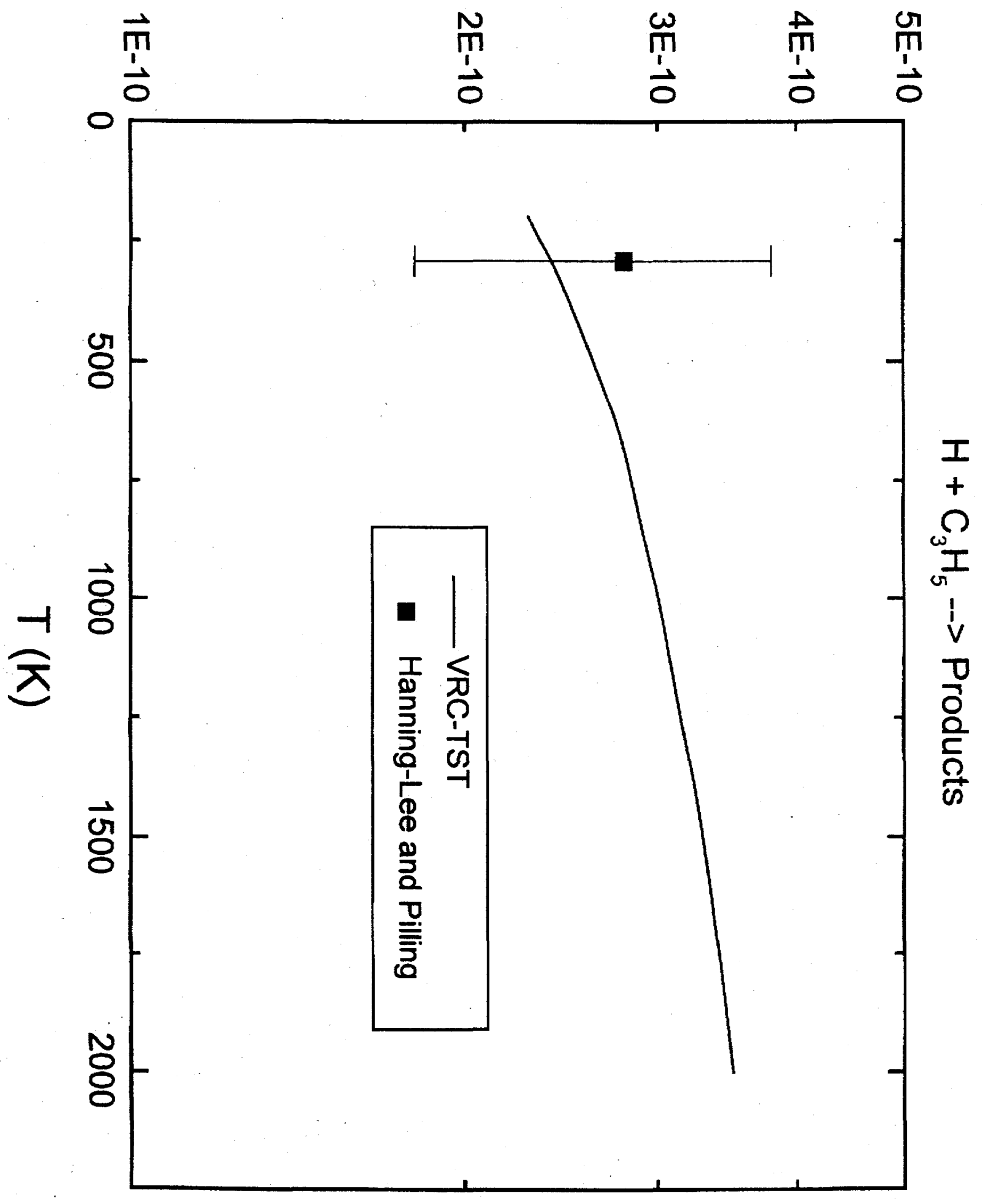




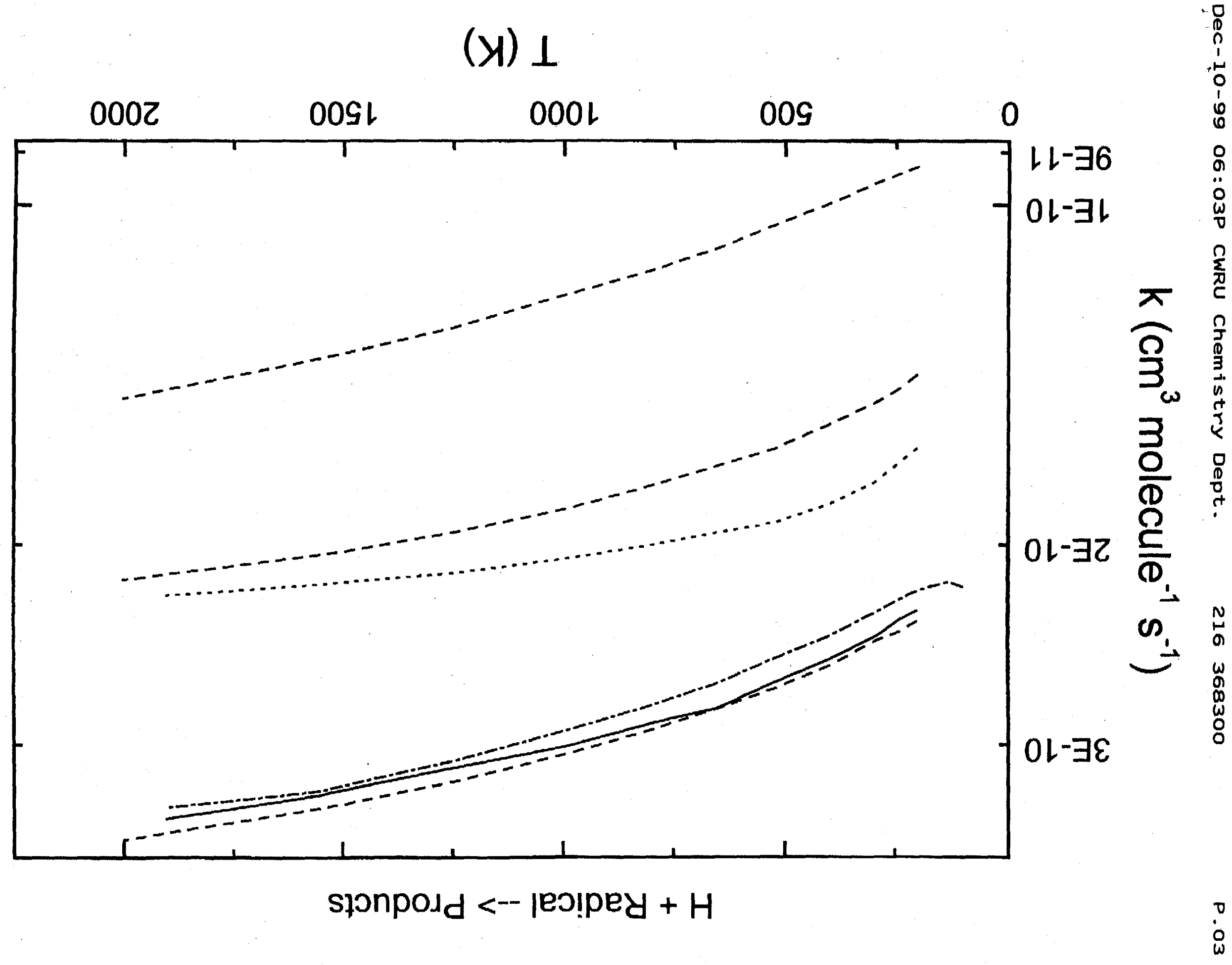

